\title{
ANÁLISE DO ACOLHIMENTO COM CLASSIFICAÇÃO DE RISCO EM UMAMATERNIDADE PÚBLICA TERCIÁRIA DE FORTALEZA
}

Raquel Amâncio Correia ${ }^{1}$, Antônia Regynara Moreira Rodrigues², Priscila França de Araújo ${ }^{3}$, Alana Santos Monte ${ }^{4}$

Objetivo: Analisar os atendimentos no acolhimento com classificação de risco em uma maternidade pública terciária de Fortaleza, Ceará. Metodologia: Estudo descritivo, retrospectivo, com abordagem quantitativa, que utilizou técnica de análise documental, com amostra de 365 instrumentos para classificação de risco, realizados no setor de emergência de uma maternidade pública terciária, no período de janeiro a junho de 2016. Resultados: A maioria (57,5\%) das mulheres foi classificada como pouco urgente para o atendimento, sendo classificadas com a cor verde. As principais queixas nos atendimentos foram: dor (34,5\%), sangramento vaginal (17,5\%) e perda de líquido (14\%). Conclusão: A clientela que busca atendimento precisa ser mais bem orientada sobre os sinais e sintomas do trabalho de parto, durante as consultas de pré-natal na atenção básica.

Descritores: Acolhimento; Enfermagem obstétrica; Emergência.

\section{ANALYSIS OF RISK CLASSIFICATION IN A TERTIARY PUBLIC MATERNITY OF FORTALEZA}

Objective: To analyze the host with risk classification in a tertiary public maternity hospital in Fortaleza, Ceará. Methodology: A descriptive, retrospective study with a quantitative approach using a documental analysis technique, with a sample of 365 instruments for risk classification, performed in the emergency sector of a tertiary public maternity hospital, from January to June, 2016. Results: The majority (57.5\%) of the women were classified as not very urgent for care being classified as green. The main complaints were pain (34.5\%), vaginal bleeding (17.5\%) and fluid loss (14\%). Conclusion: Clients seeking care need to be better informed about the signs and symptoms of labor during prenatal consultations in basic care.

Descriptors: User Embracement; Obstetric Nursing; Emergencies.

\section{ANÁLISIS DELACOGIDA CON CLASIFICACIÓN DE RIESGO EN UNA MATERNIDAD PÚBLICA TERCIARIA DE FORTALEZA}

Objetivo: Analizar la acogida con clasificación de riesgo en una maternidad pública terciaria de Fortaleza, Ceará. Metodología: Estudio descriptivo, retrospectivo, con abordaje cuantitativo, que utilizó técnica de análisis documental, con muestra de 365 instrumentos para clasificación de riesgo, realizados en el sector de emergencia de una maternidad pública terciaria, en el período de enero a junio de 2016 . Resultados: La mayoría $(57,5 \%)$ de las mujeres fue clasificada como poco urgente para la atención siendo clasificadas con el color verde. Las principales quejas en las atenciones fueron: dolor (34,5\%), sangrado vaginal (17,5\%) y pérdida de líquido (14\%). Conclusión: La clientela que busca atención necesita ser mejor orientada sobre los signos y sintomas del trabajo de parto, durante las consultas de prenatal en la atención básica.

Descriptores: Acogimiento; Enfermería Obstétrica; Urgencias Médicas.

1Escola de Saúde Pública do Ceará.

${ }^{2}$ Universidade Estadual do Ceará-UECE.

3Universidade Federal do Ceará-UFC.

${ }^{4}$ Universidade de Integração Internacional de Lusofonia Afro-Brasileira-Unilab.

Autora correspondente: Alana Santos monte. E-mail: alanasmonte@yahoo.com.br 


\section{INTRODUÇÃO}

A gestação é um processo que implica em alterações emocionais, sociais e fisiológicas no organismo da mulher, sendo considerada de baixo risco quando seu desenvolvimento não afeta desfavoravelmente a mulher e/ou o feto. Entretanto, em $20 \%$ das gestações incide uma maior probabilidade de agravos e complicações que expõem o binômio ao risco de vida, instalando-se uma gravidez de alto risco(1). No Brasil, a morte materna ainda se configura como um problema de saúde pública. Pesquisas revelam que a mortalidade materna representa apenas a ponta de um iceberg e para cada mulher que morre várias outras sofrem sequelas ou problemas crônicos de saúde ${ }^{(2)}$.

A redução da mortalidade materna é uma preocupação mundial e consta como a 5a meta a ser alcançada na "Declaração do Milênio das Nações Unidas". Uma das estratégias utilizadas pelo Ministério da Saúde/Secretaria de Vigilância em Saúde para o alcance da meta foi o lançamento do Programa da Rede Cegonha ${ }^{(3)}$. A Rede Cegonha tem por objetivos, segundo o artigo 3으 da Portaria no 1.459, 24 de junho de 2011: organizar a Rede de Atenção à Saúde Materna e Infantil para que esta garanta acesso, acolhimento e resolutividade; e reduzir a mortalidade materna e infantil com ênfase no componente neonatal(4).

No Ceará, nos últimos 17 anos analisados, observou-se uma média anual de 107 óbitos no ciclo gravídico-puerperal por causas obstétricas, com uma média de nove mortes por mês. Uma das propostas para o enfretamento da mortalidade materna, no Ceará, é a vinculação da gestante à maternidade de referência e a implantação do protocolo de Acolhimento com Classificação de Risco (ACCR) obstétrico nos hospitais( ${ }^{(3)}$.

Em obstetrícia, o acolhimento na porta de entrada dos hospitais e das maternidades é decisivo no reconhecimento de condições clínicas urgentes, necessitando de um embasamento adequado de ferramentas que subsidiem e assegurem seu processo de trabalho, utilizando para tal a classificação de risco ${ }^{(5-6)}$.

O ACCR é um dispositivo de organização dos fluxos, com base em critérios que visam priorizar o atendimento às pacientes que apresentam sinais e sintomas de maior gravidade e ordenar toda a demanda. Ele se inicia no momento da chegada da mulher, com a identificação da situação/queixa ou evento apresentado por ela. O protocolo de classificação de risco é uma ferramenta de apoio à decisão clínica e uma forma de linguagem universal para as urgências obstétricas ${ }^{(6)}$.

De acordo com a Lei do Exercício Profissional de Enfermagem, cabe ao enfermeiro realizar a Classificação de Risco, uma vez que a Lei n.. 7.498/86 incumbe privativamente ao enfermeiro, a Consulta de Enfermagem e a realização de técnicas de maior complexidade, que exijam conhecimentos científicos adequados, e a capacidade de tomar decisões rápidas ${ }^{(7)}$.

Considera-se relevante a realização de um estudo para obtenção de subsídios na melhoria da prática assistencial, mostrando a necessidade de um serviço de acolhimento com classificação de risco nas maternidades públicas, além de elencar pontos de melhoria. Por isso, faz-se necessário identificar a priorização dos atendimentos baseados em critérios obstétricos que possibilitam a melhoria da resolutividade no atendimento.

Diante do exposto, a pesquisa visa analisar os atendimentos no acolhimento com classificação de risco em uma maternidade pública terciária de Fortaleza.

\section{METODOLOGIA}

\section{Tipo de estudo}

Estudo descritivo, retrospectivo, com abordagem quantitativa, que utilizou técnica de análise documental.

\section{Participantes da pesquisa}

A população foi composta por registros impressos dos atendimentos às mulheres no setor de Emergência Obstétrica de uma maternidade pública terciária de Fortaleza. A seleção da amostra obedeceu aos seguintes critérios de inclusão: ter registrada a queixa principal apresentada pela paciente no momento da classificação de risco, ter o discriminador geral ou específico e ter a classificação de risco obtida ao final da avaliação do enfermeiro.

\section{Local do Estudo}

O estudo aconteceu em uma maternidade pública terciária de Fortaleza, referência em gestação de alto risco, que possui ACCR.

\section{Coleta de dados}

Os dados foram obtidos por meio dos registros impressos dos atendimentos na emergência que foram selecionados aleatoriamente. Foi utilizado como instrumento de coleta de dados um formulário estruturado constando itens sobre - perfil sociodemográfico das mulheres, a causa da procura ao atendimento, a cor da classificação recebida e o posterior desfecho do caso.

\section{Procedimentos de análise dos dados}

Para a organização dos dados, foi utilizado o programa SPSS versão 20.0. Foi utilizado o cálculo de amostra estimado por população finita, com grau de confiabilidade de $95 \%$ e erro amostral de $5 \%$. Portanto, a amostra do estudo foi composta por 365 fichas de atendimentos, preenchidas na emergência 
obstétrica nos meses de janeiro a junho de 2016, através de amostragem aleatória simples.

\section{Procedimentos éticos}

Foram respeitados aspectos éticos e legais relativos à pesquisa com sujeitos humanos, conforme as diretrizes regulamentadoras da resolução no 4 466, de 12 de dezembro de 2012, do Conselho Nacional de Saúde/MS. O estudo foi aprovado pelo Comitê de Ética em Pesquisa com parecer no. 1.821 .754

\section{RESULTADOS}

Com base nas fichas de atendimentos analisadas, sobre a classificação de risco, a maior parte das mulheres atendidas (57,5\%) foi classificada como pouco urgente, recebendo a cor verde como identificação; já 106 (29\%) foram classificadas como urgência, recebendo a cor amarela. Entretanto, as mulheres classificadas como emergência (cores vermelha e laranja) representaram apenas 09 (2,5\%) e 26 (7\%) atendimentos, respectivamente; classificados como não urgente, foram 15 (4\%) atendimentos.

Quanto ao perfil sociodemográfico das mulheres atendidas, observou-se que a maioria desses atendimentos era de jovens na faixa etária de 15 a 30 anos, sendo 146 (40\%) de 20 a 30 anos e 119 (32,5\%) de 15 a 19 anos; 258 (70,5\%) eram solteiras; $68 \%$ das mulheres possuiam apenas o ensino fundamental (incompleto/completo); 336 (93\%) se declararam não branca; 268 (73,5\%) eram oriundas da capital do Estado.

Acerca da condição obstétrica, observou-se que 333 (91\%) mulheres atendidas eram gestantes, sendo 168 (50,5\%) nuliparas. Quanto à idade gestacional, 232(69,5\%) estavam no 3 ㅇ trimestre quando procuraram atendimento e 137 (41\%) realizaram de 4 a 6 consultas de pré-natal (Tabela 1).

Tabela 1- Condições Obstétricas das mulheres atendidas no Acolhimento com Classificação de Risco em uma Maternidade Terciária. Fortaleza-Ce, 2016.

\begin{tabular}{|c|c|c|}
\hline Variáveis & $\mathrm{n}$ & $\%$ \\
\hline \multicolumn{3}{|l|}{ Condição obstétrica } \\
\hline Gestante & 333 & 91 \\
\hline Puérpera & 32 & 9 \\
\hline \multicolumn{3}{|l|}{ Paridade } \\
\hline Nenhum parto & 168 & 50,5 \\
\hline la 2 partos anteriores & 137 & 41 \\
\hline 3 ou mais partos & 28 & 8,5 \\
\hline
\end{tabular}

Idade gestacional

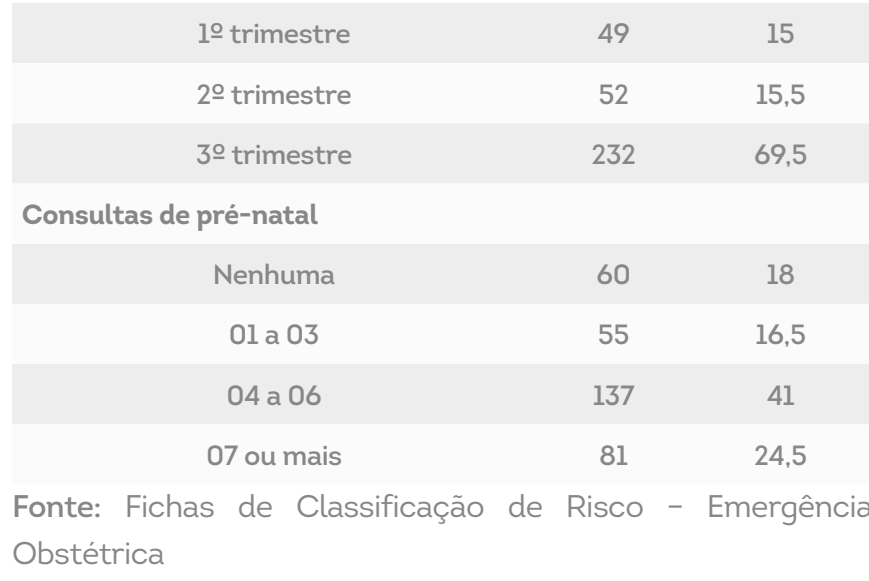

As queixas registradas no acolhimento com classificação de risco foram representadas por dor (34,5\%), seguidos de sangramento transvaginal (STV) com 63 (17,5\%) e perda de liquido 38(14\%), conforme apresenta a Tabela 2.

Tabela 2- Queixas das mulheres atendidas no Acolhimento com Classificação de Risco em Maternidade Terciária. Fortaleza-Ce, 2016.

\begin{tabular}{|ccc|}
\hline Queixas & n & $\%$ \\
\hline Dor & 126 & 34,5 \\
\hline STV & 63 & 17,5 \\
\hline Perda de Liquido/Tampão Mucoso & 50 & 14 \\
\hline Hipertensão/sinais de Iminência & 25 & 7 \\
\hline Disúria & 17 & 4,5 \\
\hline Diminuição/ausência de Movimentos & 15 & 4 \\
\hline Fetais & 12 & 3 \\
\hline Pós Datismo & 12 & 3 \\
\hline Alterações de Ultrassonografia & 45 & 12,5 \\
\hline Outras & & \\
\hline
\end{tabular}

Fonte: Fichas de Classificação de Risco - Emergência Obstétrica

No total das mulheres atendidas, apenas 89 (24,5\%) ficaram internadas na instituição, 240 (65,5\%) foram atendidas na consulta médica, sendo solicitados exames/prescrições de medicações, realizadas orientações e encaminhadas para casa, e 36 (10\%) foram transferidas para outras instituições.

Das mulheres internadas, os principais diagnósticos foram: Trabalho de Parto e Trabalho de Parto Prematuro (29\%), sindromes hipertensivas (19\%) e sindromes hemorrágicas (17\%). 
Tabela 3- Diagnósticos das mulheres que foram atendidas no Acolhimento com Classificação de Risco em Maternidade Terciária. Fortaleza-Ce, 2016

\begin{tabular}{|ccc|}
\hline Diagnósticos & n & $\%$ \\
\hline Trabalho de Parto & 26 & 29 \\
\hline Sindromes Hemorrágicas & 17 & 19 \\
\hline Sindromes Hipertensivas & 15 & 17 \\
\hline Amniorrexe Prematuro & 9 & 10 \\
\hline Diabetes & 4 & 4,5 \\
\hline Sofrimento Fetal & 4 & 4,5 \\
\hline Má- Formação Fetal & 4 & 4,5 \\
\hline Outros & 10 & 11,5 \\
\hline
\end{tabular}

Fonte: Fichas de Classificação de Risco - Emergência Obstétrica

\section{DISCUSSÃO}

Foi realizada avaliação da classificação de risco que permite à equipe atribuir um grau de prioridade clinica para esse atendimento, assim como, o máximo de tempo que pode esperar conforme a prioridade identificada, ocorrendo de acordo com a gravidade de risco e baseado em cores: Azul (não urgente atendimento em até 240 minutos); Verde (pouco urgente: consulta médica em até 120 minutos); Amarelo (urgente: consulta médica priorizada em até 30 minutos, mantendo reavaliação periódica); Laranja (muito urgente: atendimento médico no prazo máximo de 10 minutos); Vermelho (prioridade máxima: emergência, centro obstétrico ou atendimento médico imediato)(6).

Observou-se que a maior demanda do serviço foi destinada aos atendimentos com pouca urgência, demonstrando fragilidade da atenção primária, principalmente no quesito pré- natal, já que a justificativa ou queixa para estes atendimentos eram: dor relacionada ao trabalho de parto, perda de liquido, sintomas clínicos, amenorreia e retorno para mostrar exames. Sendo este quadro determinante para a superlotação do serviço, pois as pacientes que deveriam estar sendo atendidas nas unidades de atenção primária à saúde estão gerando, desnecessariamente, uma maior demanda nos hospitais.

Embora haja evidências da melhoria da cobertura da assistência pré-natal no Brasil, existem ainda questões relacionadas à dificuldade de acesso, à baixa qualidade da atenção pré- natal, carência de orientações às gestantes, início tardio da assistência pré-natal e o baixo número de consultas, bem como a falta de profissionais aptos e treinados para o bom atendimento da mulher, que comprometem a qualidade da assistência durante o pré-natal e expõem mulher na gestação, no parto e puerpério a riscos e consequências adversas $^{(8)}$.
É comum observar uma emergência obstétrica superlotada com mulheres que apresentam diversos tipos de situações que não são necessariamente casos urgentes ou de emergência, sendo observados casos que poderiam ser facilmente resolvidos através do atendimento ambulatorial ou até mesmo por meio da assistência primária(9,10)

Corroborando com os achados, em estudo realizado em outra maternidade terciária de Fortaleza, 48,3\% das mulheres foram classificadas como pouco urgente, $22,3 \%$ classificadas como não urgente e 18,8\% classificadas como urgente ${ }^{(11)}$. No âmbito internacional, em revisão sistemática de 31 estudos realizados em vários países, foi constatado que a prevalência do uso inadequado dos serviços de emergências apresentou variação de $20 \%$ a $40 \%{ }^{(12)}$.

Quanto aos dados sociodemográficos, a investigação mostra um percentual significativo de gestantes adolescentes (39,5\%), evidenciando uma realidade do país, apesar de recente pesquisa mostrar que a gravidez na adolescência teve uma queda de $17 \%$ no Brasil( ${ }^{(13)}$. Além disso, percebese baixo nível educacional dessas mulheres, que, aliado ao medo e ao sentimento de insegurança, podem influenciar no desconhecimento dos sinais e sintomas de risco que realmente necessitam da busca de uma unidade terciária.

A elevada porcentagem das mulheres residentes na capital e região metropolitana advém do fato de estarem mais próximas da Maternidade, e desta ser uma maternidade de referência. No entanto, chama atenção também o número de atendimentos de mulheres procedentes dos municípios do interior do Estado. Isso provavelmente reflete a precariedade da assistência prestada nessas localidades e a necessidade de melhorias, que podem ser alcançadas com a organização de uma assistência regional e a descentralização do atendimento de pré- natal e parto de alto risco, fornecendo a essas regiões equipamentos, exames complementares, medicamentos e profissionais especializados.

Os atendimentos realizados na emergência da maternidade tiveram como público alvo as gestantes, nuliparas, no terceiro trimestre. Esse fato pode ser explicado pela falta de experiência dessas mulheres, aliado à ansiedade do final da gestação. No entanto, ainda houve atendimentos que deveriam ser realizados nos serviços da atenção primária ou atenção secundária, tais como: fluxo menstrual intenso, dificuldade na amamentação e teste de gravidez.

As principais queixas apresentadas pelas mulheres atendidas no setor de acolhimento com classificação de risco foram dor (34,5\%), STV (17,5\%) e perda de liquido (14\%). Estudos realizados em maternidades de referência no Rio de Janeiro e Ceará mostraram que as causas de procura do atendimento também foram em sua maioria por dor, STV e perda de liquido(11,14). Essas queixas podem representar um 
sinal de trabalho de parto ou uma complicação obstétrica.

Os principais diagnósticos encontrados para internamento foram representados pelas sindromes hipertensivas e sindromes hemorrágicas, que aparecem somente após a metade do 2 ㅇ trimestre da gestação, agravando-se no 3ㅇ trimestre. As sindromes hipertensivas e as sindromes hemorrágicas estão entre as principais causas de óbito materno por causa direta ${ }^{(2)}$. Dentre as morbidades, tem destaque a sindrome hipertensiva, que pode resultar em altos índices de prematuridade e retardo do crescimento fetal. A pré-eclâmpsia é indicativa de urgência, pois, se não for controlada, pode evoluir para a eclâmpsia, caracterizada pela presença de convulsões tônico-clônicas generalizadas, podendo causar morte materna e fetal ${ }^{(15)}$.

Já as Síndromes Hemorrágicas, presentes na segunda metade da gestação, constituem-se em frequentes diagnósticos em obstetrícia. Destacam-se como uma das principais causas de internação de gestantes no periodo anteparto, com importante aumento da morbimortalidade materna e perinatal, assim como de partos operatórios. Entre as causas obstétricas mais frequentes, têm-se o descolamento prematuro de placenta e a placenta prévia(16).

Nesse sentido, percebe-se a importância da captação precoce da gestante e do acompanhamento pré- natal de qualidade, assim como da assistência hospitalar adequada. Portanto, enfatizam-se as ações secundárias essenciais no processo de redução dos óbitos maternos. Com isso evidencia-se a importância dos serviços de acolhimento com classificação de risco nas maternidades, para a triagem dessas pacientes, com a realização de intervenções imediatas.

\section{Limitações do estudo}

O estudo realizado apresentou limitações por ter sido desenvolvido em uma única maternidade e pelo reduzido tamanho da amostra.

\section{Contribuições para a prática}

Como contribuições a prática da saúde e à enfermagem, ressaltam-se que as informações discutidas como condições gestacionais, queixas das pacientes e principais diagnósticos encontrados durante o acolhimento são fundamentais para avaliar a acurácia da utilização dos protocolos de ACCR, na busca por resultados favoráveis à qualidade do atendimento em serviços de emergência.

\section{CONCLUSÃO}

Observou-se que o perfil de maior prevalência não foi os dos atendimentos de urgência e emergência e sim de atendimentos com pouca urgência e sem urgência. Com isso mostrou-se a fragilidade existente no atendimento destas mulheres na atenção primária, quanto às orientações corretas da necessidade real da busca de um atendimento especializado, como atendimento de urgência e emergência em uma maternidade, principalmente às gestantes.

O estudo mostrou que o atendimento em uma emergência obstétrica com a utilização do protocolo de acolhimento com classificação de risco contribui para a instituição, por proporcionar um atendimento que possibilita a identificação dos riscos obstétricos, podendo melhorar o fluxo de atendimento e diminuir a superlotação das maternidades, uma vez que os casos não urgentes podem ser facilmente identificados e encaminhados para o serviço ambulatorial ou da atenção primária.

\section{Contribuição dos autores}

Raquel Amâncio Correia contribuiu com a Concepção e/ ou desenho, análise e interpretação dos dados e redação do artigo; Antônia Regynara Moreira Rodrigues contribuiu com a revisão crítica; Priscila França de Araújo contribuiu com Concepção e/ou desenho e redação do artigo; Alana Santos Monte contribuiu com a redação do artigo, revisão crítica, revisão final. 


\section{REFERÊNCIAS}

1. Rodrigues ARM, Dantas SLC, Pereira AMM, Silveira MM, Rodrigues DP. Gravidez de alto risco: análise dos determinantes de saúde. Sanare. 2017; 16 Suppl 01:23-8.

2.Monte AS, Teles LMR, Costa CC, Gomes LFS, Damasceno AKC. Análise das condições potencialmente ameaçadoras de vida de mulheres em unidade de terapia intensiva. Rev Rene. 2017 jul-ago; 18(4):461-7.

3. Secretaria de Saúde do Estado do Ceará (CE). Informe Epidemiológico Mortalidade Materna. 2015

4.Ministério da Saúde (BR). Manual Prático para Implementação da Rede Cegonha. Brasília: Ministério da Saúde, 2011.

5. Hedlund ACB, Ilha CB, Hoffmann IC, Krusche JB, Pimenta LF , Braz MM.Percepção de profissionais sobre acolhimento com classificação de risco no centro obstétrico. Saúde (Santa Maria). 2015 Jul./Dez; 4l(2):149-60.

6. Ministério da Saúde (BR). Secretaria de Atenção à Saúde. Departamento de Ações Programáticas Estratégicas. Manual de acolhimento e classificação de risco em obstetricia- Brasilia: Ministério da Saúde, 2014

7. Maranha NB, Silva MCA, Brito IC. A consulta de enfermagem no cenário da atenção básica e a percepção dos usuários: revisão integrativa. Academus Revista Científica da Saúde. 2017 jan./abr.; 2(1): 45- 52 .

8.Costa CSC, Vila VSC, Rodrigues FM, Martins CA, Pinho LMO Caracteristicas do atendimento pré-natal na Rede Básica de Saúde. Rev. Eletr. Enf. [Internet]. 2013 [Citado 2017 jul 10] abr/ jun;15(2):516-22. Disponivel em: https://www.fen.ufg.br/fen_revista/v15/n2/pdf/v15n2a26.pdf

9. Hedlund ACB, Ilha CB, Hoffmann IC, Krusche JB, Pimenta LF, Braz MM. Percepção de profissionais sobre acolhimento com classificação de risco no centro obstétrico. Saúde (Santa Maria). 2015; 41(2):149-60
10. Monteiro MM, Sá GGM,Oliveira Neto JG, Lopes KDCL, Carvalho DAC, Martins MCC.Emergências obstétricas: caracteristicas de casos atendidos por serviço móvel de urgência. R. Interd. 2016 abr. mai. jun.; 9(2):136-44.

11. Brilhante AF, Vasconcelos CTM, Bezerra RA, Lima SKM, Castro RCMB, Fernandes AFC. Implementação do protocolo de acolhimento com classificação de risco em uma emergência obstétrica. Rev Rene. 2016 jul-ago; 17(4):569-75.

12.Carret MLV, Fassa AG, Domingues MR. Inappropiate use of emergency services: a systematic review of prevalence and associated factors. Cad Saúde Pública. 2009; 25(1)7-28.

13.Valadares C. Gravidez na adolescência tem queda de $17 \%$ no Brasil. 2017. Disponivel em: https://www.portalsaude.saude.gov. br. Acesso em 01/06/2017

14.Pereira ALF, Lima AEF. Acolhimento com classificação de risco em uma maternidade publica. Rev enferm UFPE on line. 2014; 8(supl. 1):2309-15.

15. Oliveira GS, Paixão GPN, Fraga CDS, Santos MKR, Santos MA. Assistência de enfermeiros na sindrome hipertensiva gestacional em hospital de baixo risco obstétrico. Rev Cuid [Internet]. 2017 Dec [citado 2018 Feb 06] ; 8( 2 ): 1561-1572. Disponivel em: http://www.scielo.org.co/scielo.php?script=sci_ arttext\&pid=\$2216-097320170002015618lng=en. http://dx.doi. org/10.15649/cuidarte.v8i2.374.

16.Lugo SAM. Caracterización de las pacientes con hemorragia uterina puerperal en las que se utilizó misoprostol. Rev Cubana Obstet Ginecol [Internet]. 2014 Jun [citado 2018 Feb 06] ; 4O( 2 ): 145-154. Disponible en: http://scielo.sld.cu/scielo.php?script=sci_arttextEpid=S0138-600X20140002000028lng=es. 